\title{
Encore la Gorgone
}

The return of the Gorgon

\section{Françoise Frontisi-Ducroux}

\section{(2) OpenEdition}

\section{Journals}

Édition électronique

URL : http://journals.openedition.org/imagesrevues/8852

DOI : 10.4000/imagesrevues.8852

ISSN : 1778-3801

\section{Éditeur :}

Centre d'Histoire et Théorie des Arts, Groupe d'Anthropologie Historique de l'Occident Médiéval, Laboratoire d'Anthropologie Sociale, UMR 8210 Anthropologie et Histoire des Mondes Antiques

\section{Référence électronique}

Françoise Frontisi-Ducroux, « Encore la Gorgone », Images Re-vues [En ligne], Hors-série 9 | 2020, mis en ligne le 01 décembre 2020, consulté le 16 mars 2021. URL : http://journals.openedition.org/ imagesrevues/8852 ; DOI : https://doi.org/10.4000/imagesrevues.8852

Ce document a été généré automatiquement le 16 mars 2021.

Images Re-vues est mise à disposition selon les termes de la Licence Creative Commons Attribution Pas d'Utilisation Commerciale 4.0 International. 


\section{Encore la Gorgone}

The return of the Gorgon

Françoise Frontisi-Ducroux

L'iconicité constitutive de la figure de Méduse 
1 Dans son petit ouvrage intitulé Peri tou oikou, diversement traduit par Sur un appartement, ou encore La salle, Lucien de Samosate décrit une galerie de peintures. L'un des tableaux représente Persée décapitant Méduse. Selon le procédé de l'ekphrasis, l'auteur rappelle l'histoire qui est figurée :

«On voit Persée ..., Méduse qui se fait couper la tête, et Athéna qui protège Persée. Il a osé son exploit, mais il n'a pas vu son acte, si ce n'est le reflet de la Gorgone sur le bouclier. Car il connaît le prix à payer pour la vue de la réalité. ${ }^{1}$ "

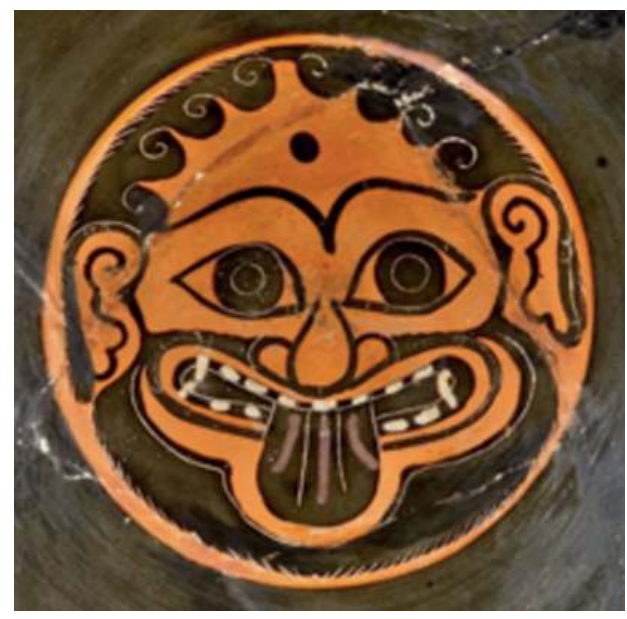

2 Quelques siècles plus tôt Apollodore en racontant l'histoire avait lui aussi précisé :

" c'est en se détournant et en regardant sur le bouclier de bronze grâce auquel il a vu le reflet de la Gorgone, qu'il l'a décapitée. ${ }^{2}$ »

3 Les deux écrivains soulignent que le héros n'a pu voir de la Gorgone qu'une eikon, en l'occurrence son reflet, sur le bouclier d'Athéna obligeamment orienté par la déesse pour fonctionner comme un miroir. L'impossibilité de contempler la face de Méduse est la composante intrinsèque du mythe. La voir et en être vu c'est mourir dans l'instant, pétrifié par son pouvoir fatal.

4 Facile à dire. Mais comment le montrer puisque l'image en est interdite ? Comment les artistes, techniciens du figuratif, se sont-ils tirés de ce dilemme ? Ou plus exactement, puisque la figure de la Gorgone est partout présente dans l'environnement des Grecs, faisant l'objet d'images multiples, quels procédés les créateurs d'images ont-ils mis en œuvre pour signifier leur conscience de cet interdit visuel, constamment signalé verbalement, en dénonçant eux aussi l'iconicité constitutive de la figure de Méduse ?

\section{Une série de vases de la peinture italiote}

$5 \quad \mathrm{Au} \mathrm{IV}^{\mathrm{e}}$ siècle, la peinture italiote propose une version narrative, à peu près parallèle à ce que disent les textes. À peu près seulement car les artistes ont choisi un épisode différent de la version textuelle. Une série de vases montre en effet Athéna faisant voir à Persée le reflet de la tête coupée. Après la décapitation donc. 
Figure 1

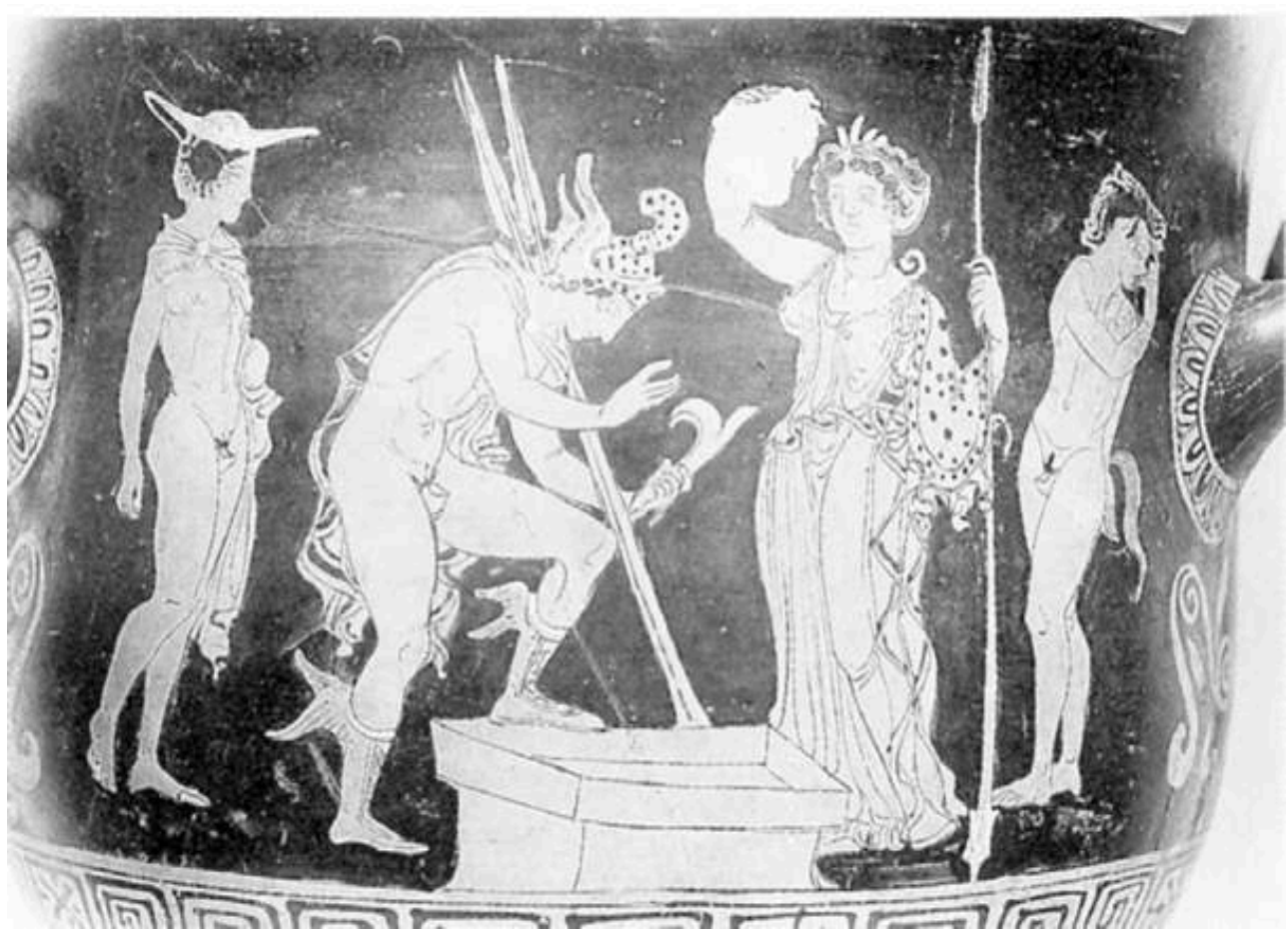

Cratère apulien à figures rouges, Leipzig, Université T 83.

6 Ainsi d'un cratère apulien ${ }^{3}$ (fig. 1 ) sur lequel Athéna brandit la tête qu'elle regarde. Persée, penché au-dessus d'un puits, fait de la main un geste de surprise signifiant qu'il voit la tête reflétée. Qui ne nous est pas visible. Derrière Athéna un satyre se détourne, marquant l'interdit visuel avec force, car le satyre est réputé pour son appétit scopique $^{4}$. Méduse est bien présente, offerte au spectateur du vase comme, dans l'image, aux spectateurs divins, Hermès et Athéna. Sa nature purement iconique pour les autres protagonistes, Persée et le satyre, n'est que suggérée par la mise en scène. 
Figure 2

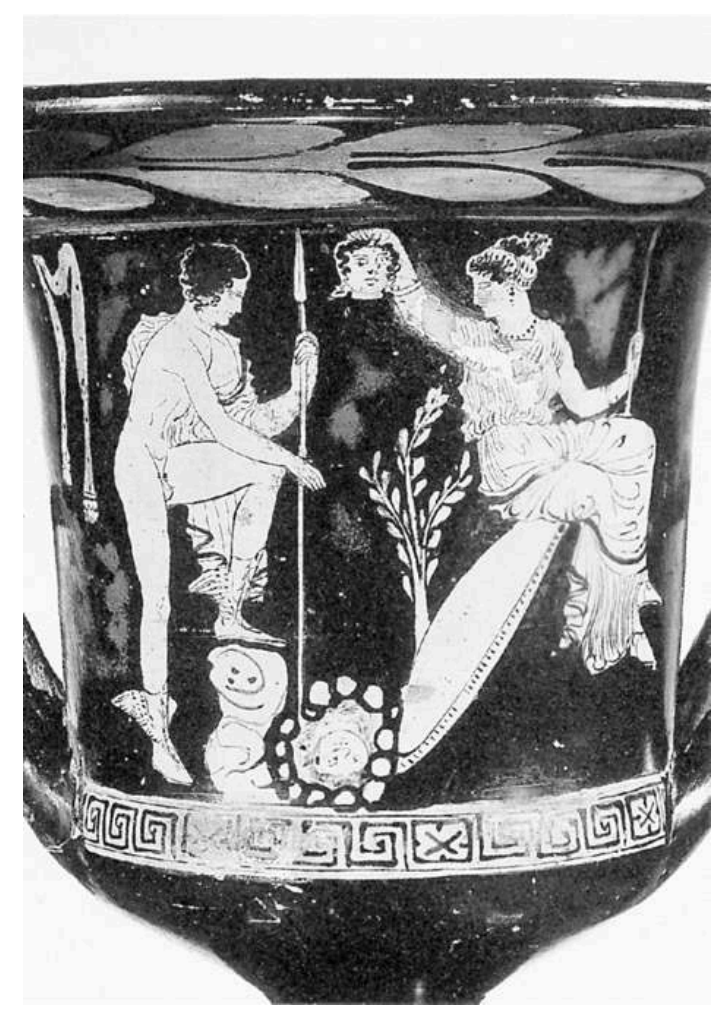

Cratère apulien à figures rouges, Gotha, Schlossmuseum AHV 72.

7 Sur un autre cratère ${ }^{5}$ (fig. 2), le reflet de la tête est nettement visible sur l'eau d'une mare circulaire, entourée de gros cailloux. Les traits de Méduse sont bien dessinés et leur reflet canoniquement inversé. Athéna est perchée sur son bouclier, de profil, vraisemblablement appuyé contre un rocher. Les deux protagonistes orientent leurs regards vers le reflet, que Persée indique de la main. Sur le bouclier on peut entrevoir, très floue, une esquisse de décor. 
Figure 3

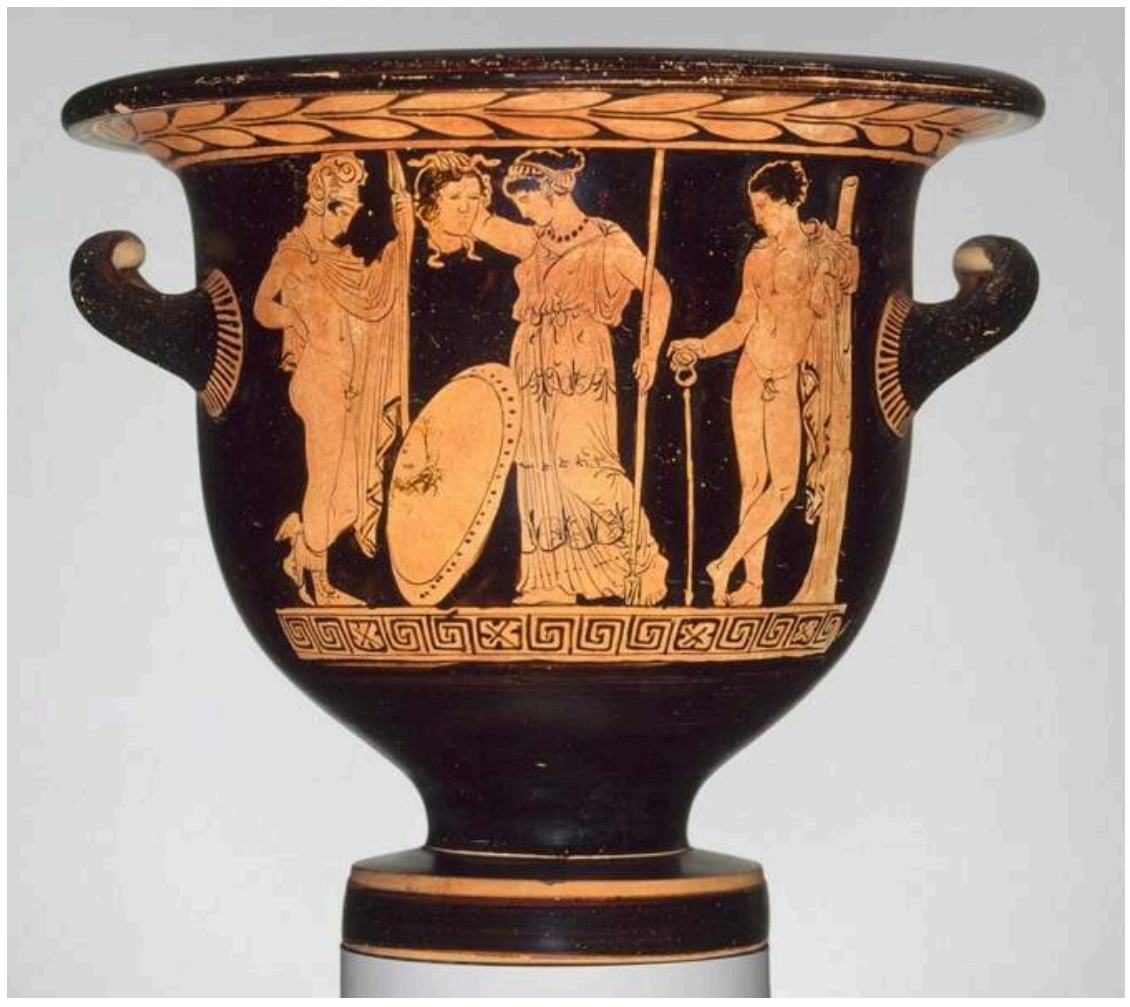

Cratère apulien à figures rouges, Boston, Museum of Fine Arts 1970.237.

8 Sur le cratère apulien de Boston ${ }^{6}$ (fig. 3) la mise en scène est différente. Athéna brandit la tête, en trois-quarts/face, au-dessus du bouclier dressé contre son corps, et trois personnages regardent le reflet, correctement inversé et légèrement coupé par l'orbe du bouclier : encadrant Athéna, Persée et Hermès affichent la pose de la contemplation. La pose de Persée. Comme sur l'image précédente la composition superpose en une ligne verticale la vraie tête (non regardée mais visible du spectateur) et son reflet regardé.

Figure 4

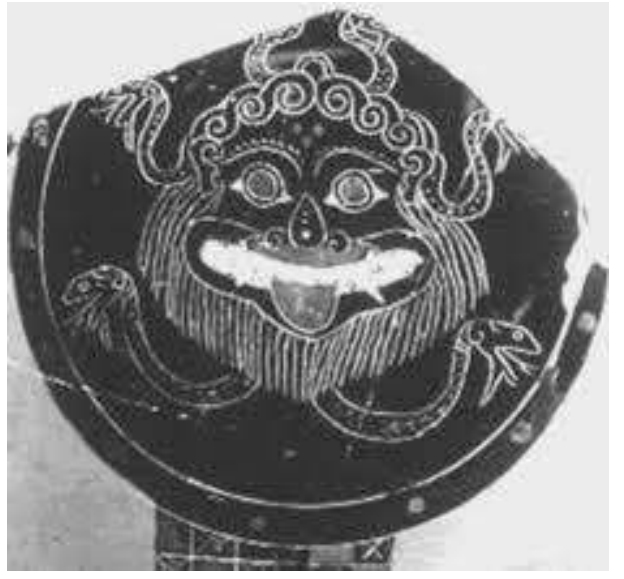

Amphore à col à figures noires, Boston Museum of Fine Arts 01.8026 (détail). 
Le reflet se trouve ici à la place occupée traditionnellement par le gorgoneion qui décore souvent le bouclier de la déesse, comme on peut le voir, entre autres, sur un fragment attribué au peintre d'Amasis ${ }^{7}$ (fig. 4).

Figure 5

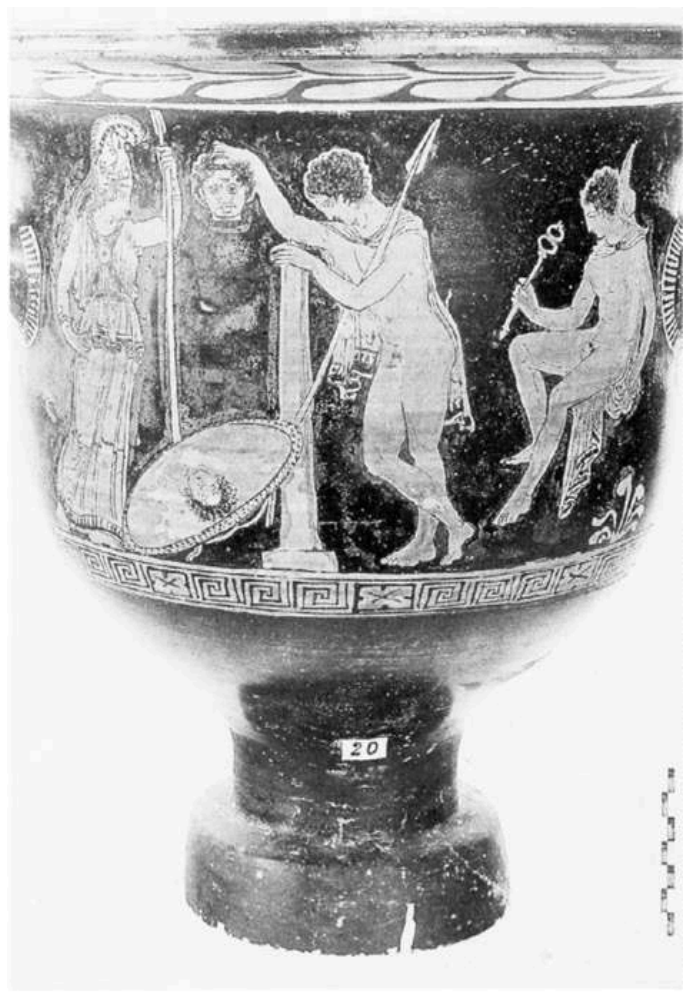

Cratère en cloche apulien à figures rouges, Ruvo, Museo Jatta 20.

10 Sur un cratère en cloche de Ruvo ${ }^{8}$ (fig. 5), c'est Persée lui-même, entre Athéna et Hermès, qui élève la tête au-dessus du bouclier; mais celui-ci est couché à l'envers et c'est l'extérieur de l'orbe qui renvoie le reflet. Ici encore la posture de la contemplation est soulignée : Hermès assis sur un rocher, Athéna appuyée sur sa lance, et Persée main sur un pilier.

\section{La valeur iconopoiétique de la scène du reflet}




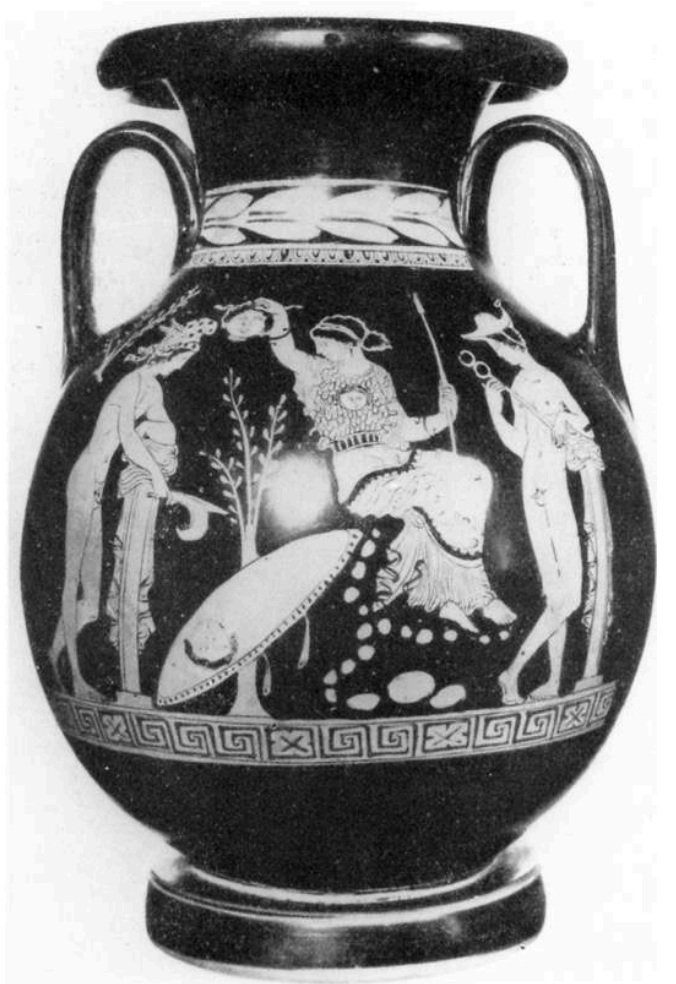

Péliké apulienne à figures rouges, Tarante, collection privée.

11 Une autre formule apparaît sur une péliké apulienne de Tarente $^{9}$ (fig. 6). La composition est analogue mais enrichie d'un troisième élément. Les trois protagonistes contemplent le reflet sur le bouclier, mais Athéna qui, assise sur un rocher, au centre, soulève la tête de Méduse, porte sur son égide un gorgoneion, position également canonique et conforme à la tradition textuelle. La tête coupée est donc montrée au spectateur du vase en triple exemplaire: la vraie, brandie par la déesse, qui dans l'espace iconique n'est pas regardée. Le reflet inversé, entièrement visible et regardé par les protagonistes. Et le gorgoneion qui, selon la vulgate, est la vraie tête, remise après la décapitation par Persée à sa protectrice et devient un motif figuratif fréquent. Les trois exemplaires ne sont pas stéréotypés, mais cherchent à produire un effet de « vivant ». Les trois sont offerts de face, à la vue du spectateur. Loin d'accuser le peintre d'incohérence, il faut comprendre son intention de montrer en trois espèces la tête de la Gorgone, voire d'indiquer de façon ludique que pour un spectateur hors espace iconique les trois têtes ne sont que des eikones, dès lors qu'elles lui sont regardables.

12 Le jeu sur les différents sens du mot eikon est un topos, qui peut s'enrichir comme le fait Philostrate, à propos d'un tableau représentant Narcisse, d'un déploiement des valeurs de la notion de graphein, activité picturale, entre autres :

« La source (dé)peint Narcisse et la peinture (dé)peint la source et tout ce qui a trait

à Narcisse. ${ }^{10}$ »

13 Les peintres italiotes eux aussi savent montrer qu'ils sont conscients de « peindre » ce que « peignent » la source ou le bouclier. 
Figure 7

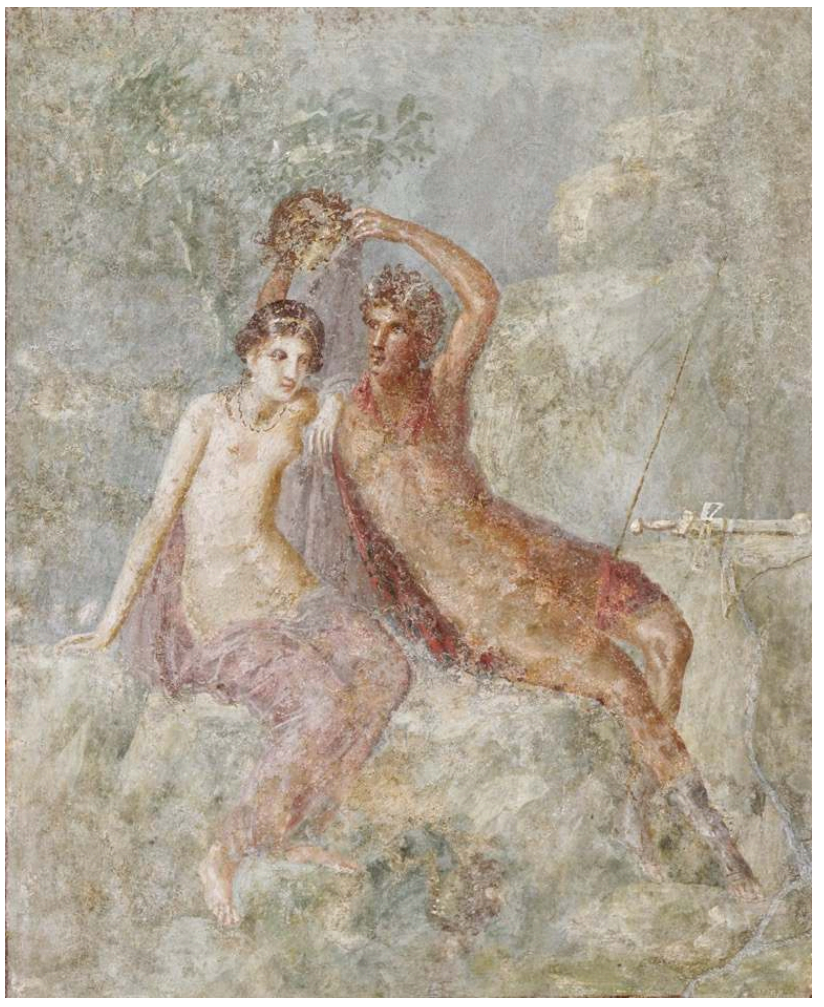

Peinture murale, Naples, Museo Nazionale 8996 (Pompei VII,4,51 et 31).

Figure 8

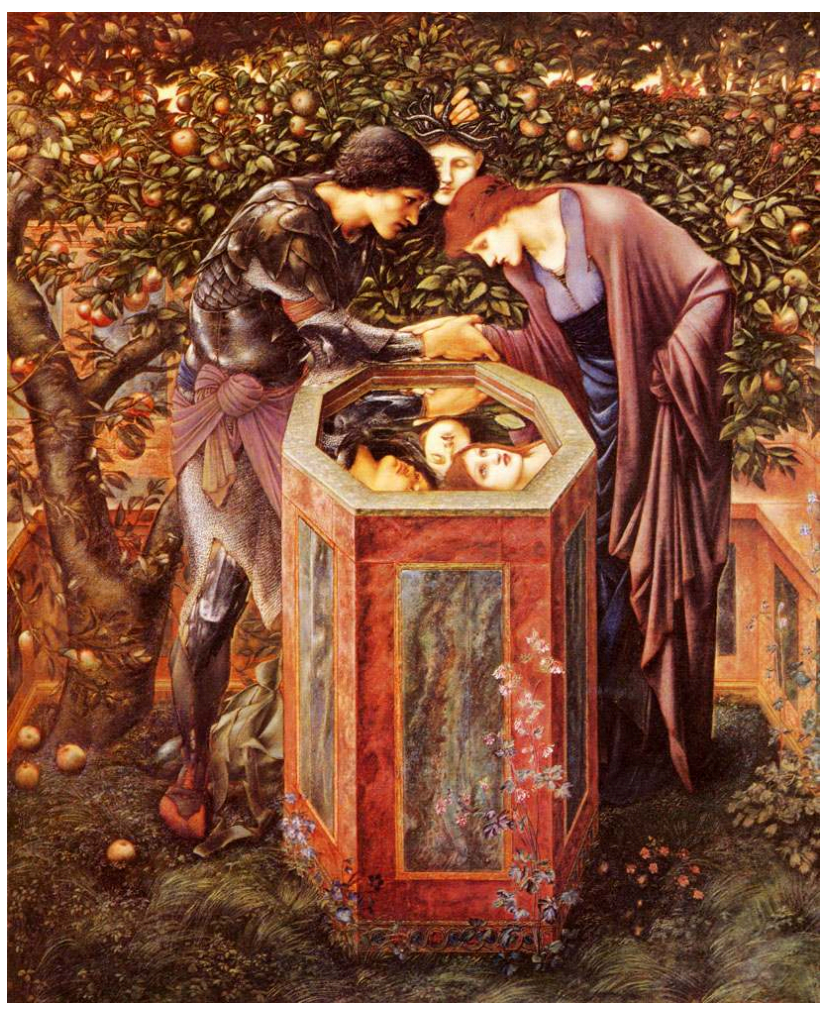

Burne Jones, The Baleful Head (Le cycle de Persée). 

confirmée par son succès. Depuis la peinture romaine (fig. 7) jusqu'à, par exemple, Burne Jones, dans son Cycle de Persée ${ }^{11}$ (fig. 8). L'un des tableaux montre Persée, qui, stimulé par l'invention d'Athéna, imite la déesse en montrant à son tour la tête reflétée, à son Andromède, délivrée d'un autre monstre. mettent en image une histoire, qui parallèlement peut se raconter et qu'elles évoquent. Cependant l'interdit visuel constitutif de la face de Méduse a suscité d'autres œuvres plus spécifiquement figuratives. Et ce très tôt, en peinture attique dès le début de la figure rouge au ve siècle.

\section{Les Gorgones du peintre de Pan, penseur figuratif}

L'imagier connu sous le nom de peintre de Pan peut être qualifié de penseur figuratif. Plusieurs de ses œuvres témoignent d'une conscience du problème posé par la représentation de la tête gorgonéenne.

Figure 9

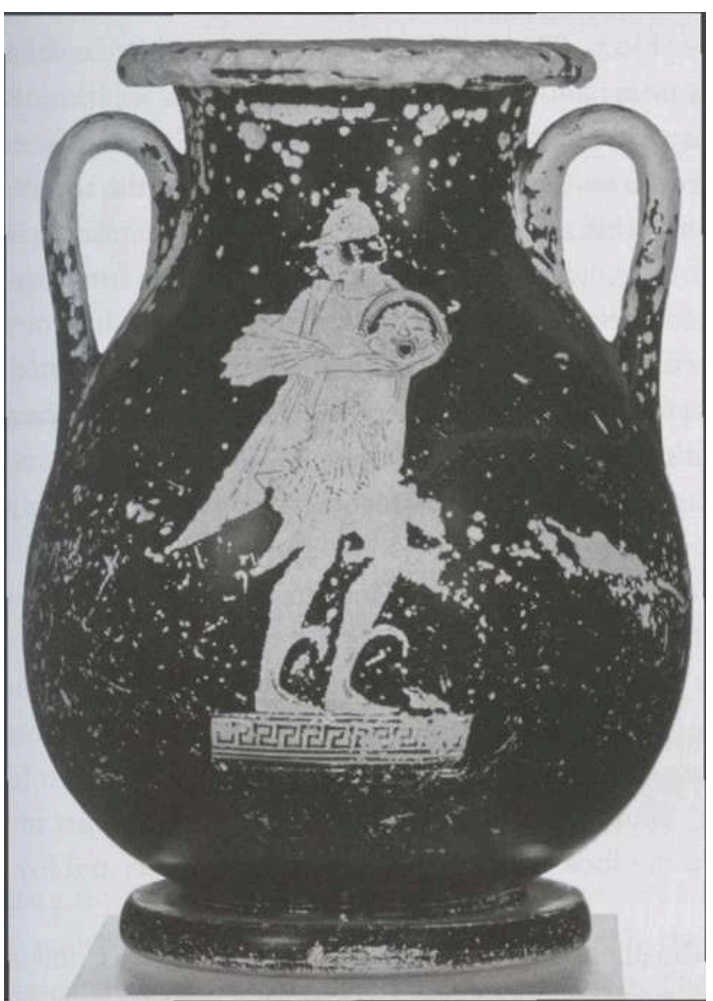

Péliké à figures rouges, Munich, Antikensammlungen 8725

Sur une péliké ${ }^{12}$ (fig. 9) Persée est représenté seul portant devant lui la tête coupée. Plaquée sur sa poitrine elle occupe à peu près la place du gorgoneion sur l'égide d'Athéna. Et le héros, bien qu'il ne risque pas de voir la face placée devant lui, détourne son visage "canoniquement" comme il le fait sur les scènes de décapitation. Cette image novatrice travaille avec des éléments traditionnels : le geste de l'apostrophé détournement intra-iconique avec exhibition au spectateur hors image, et le gorgoneion 
affiché sur la poitrine d'Athéna. Deux façons d'exprimer la nature non visible de la Gorgone, sinon en image. Le revers du vase représente un aulète, allusion à l'invention de cet instrument par Athéna, pour mimer en musique l'horreur de la décapitation. L'aulos et ses airs spécifiques constituent le doublet sonore de la face de Méduse, son eikon musicale.

Cette composition très dense fait certes allusion à une narration mais de façon synthétique, par des procédures purement figuratives.

Figure 10

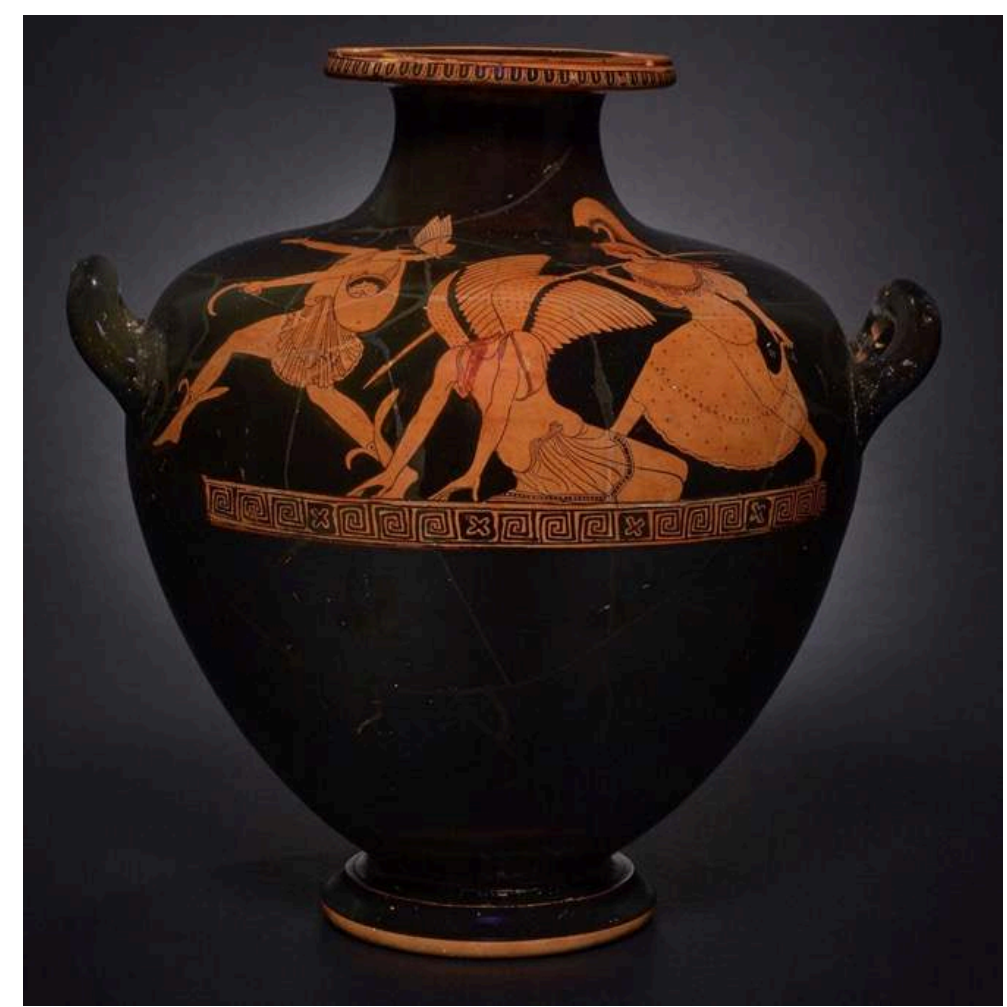

Hydrie à figures rouges, Londres, British Museum E 181.

Sur l'hydrie de Londres ${ }^{13}$ (fig. 10) le principe est analogue. Persée s'enfuit avec la tête coupée émergeant de la besace. Le héros regarde en arrière, détourné par rapport à la harpé qu'il tend en avant. Le schème traditionnel de la décapitation "à tâtons " (cf. infra fig. 12, 13,14) est transformé en retournement vers le corps affaissé de Méduse, enfin contemplable, comme l'indique le regard (avide) de Persée au-dessus du cou coupé, dégoulinant de sang. Athéna accourt, légère, pour confirmer son œuvre : faire voir ce qui ne se peut voir, en l'occurrence une vacuité. Si la scène est narrative elle choisit un moment peu représenté : l'instant d'après la décollation. Persée ne voit qu'une absence. La tête n'est visible que du spectateur, mais à moitié seulement et ses yeux sont à demi fermés. Humour d'un peintre qui se joue de l'interdit visuel et de sa représentation. 
Figure 11

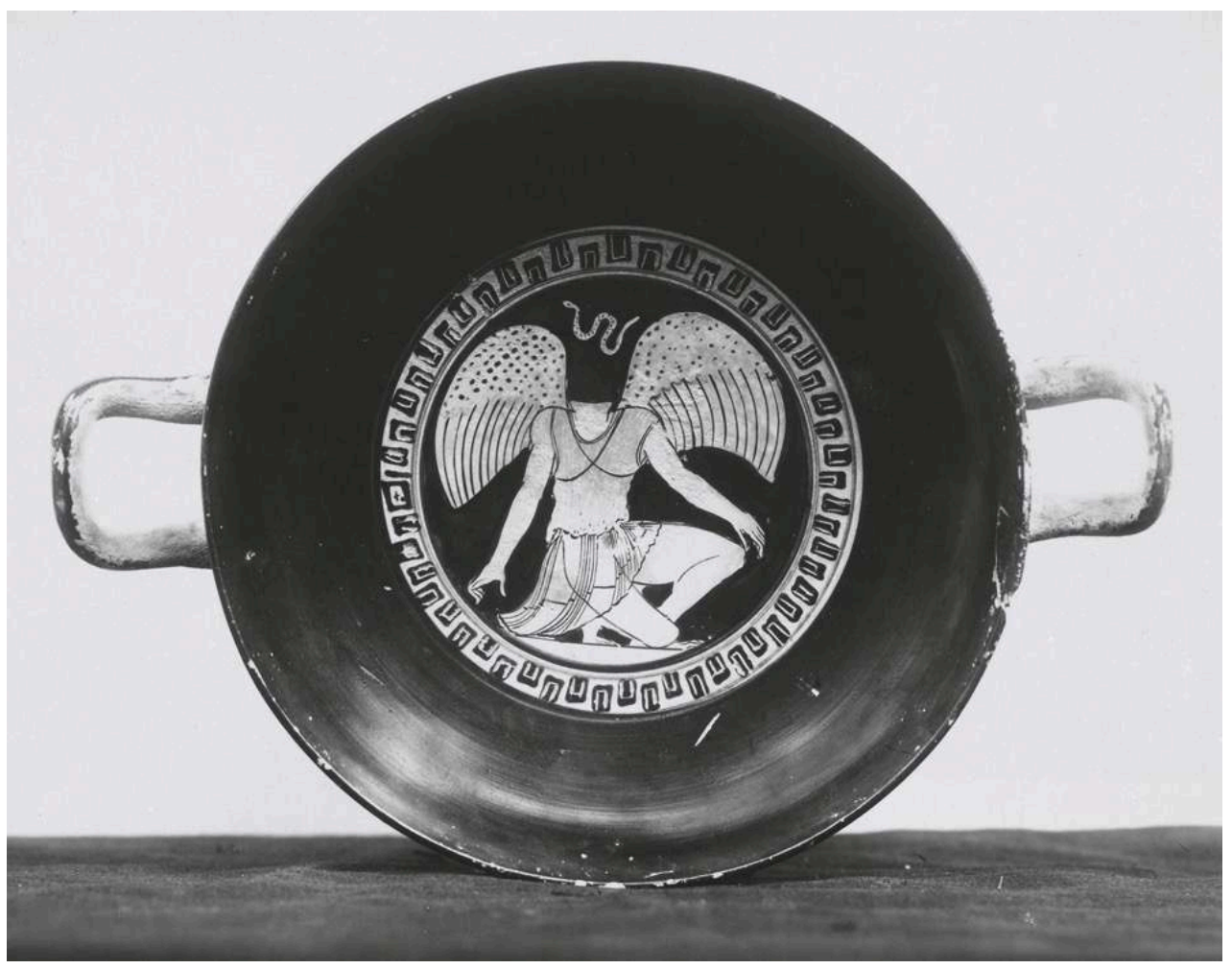

Coupe à figures rouges, Berlin, Antikensammlung 4951.

20 Toujours du même, une figure saisissante en fond de coupe ${ }^{14}$ (fig. 11). Une Méduse décapitée, seule, ce qui est exceptionnel, car les scènes qui suivent la décapitation la montrent accompagnée de ses sœurs qui s'élancent à la poursuite de Persée. Elle s'effondre, corps de face, en position semi agenouillée, traditionnelle. Entre les deux ailes qui encadrent le cou proprement tranché, un petit serpent se tortille. Traditionnellement les serpents participent à la poursuite du meurtrier. Celui-ci, unique séquelle, délimite la béance, faisant voir le "sans-tête », ellipse de l'eikon dans l'image de ce peintre du décalage.

\section{Encore la Gorgone, du modèle aux succédanés en image}

Quelques images, antérieures ou contemporaines, feront mieux voir ce dont le peintre de Pan se démarque, dans sa réflexion figurative. 
Figure 12

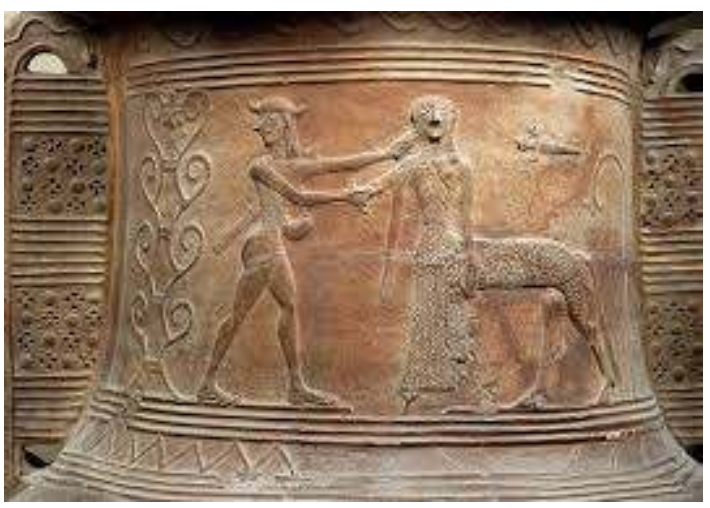

Pithos orientalisant à reliefs, Paris, Musée du Louvre CA 795.

Figure 13

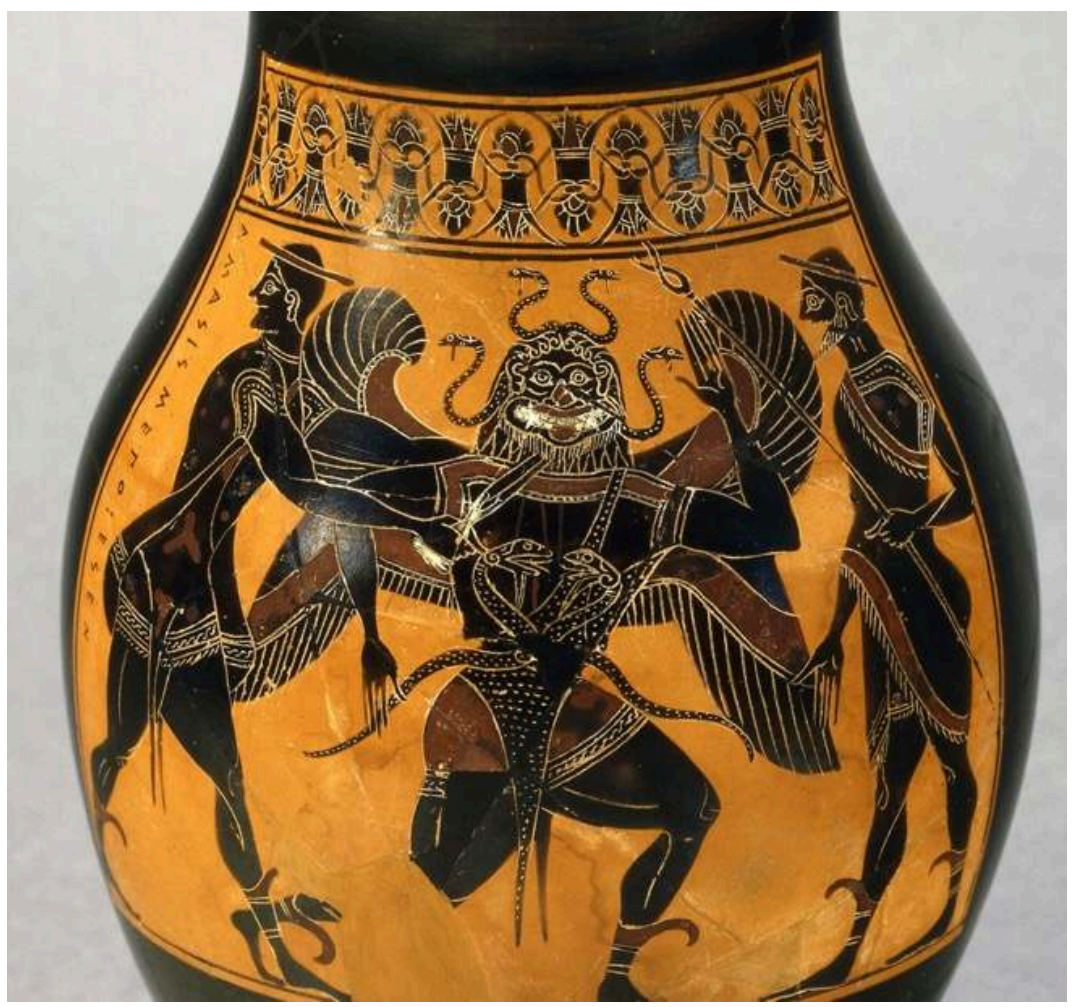

Olpé à figures noires, Londres, British Museum B 471. 
Figure 14

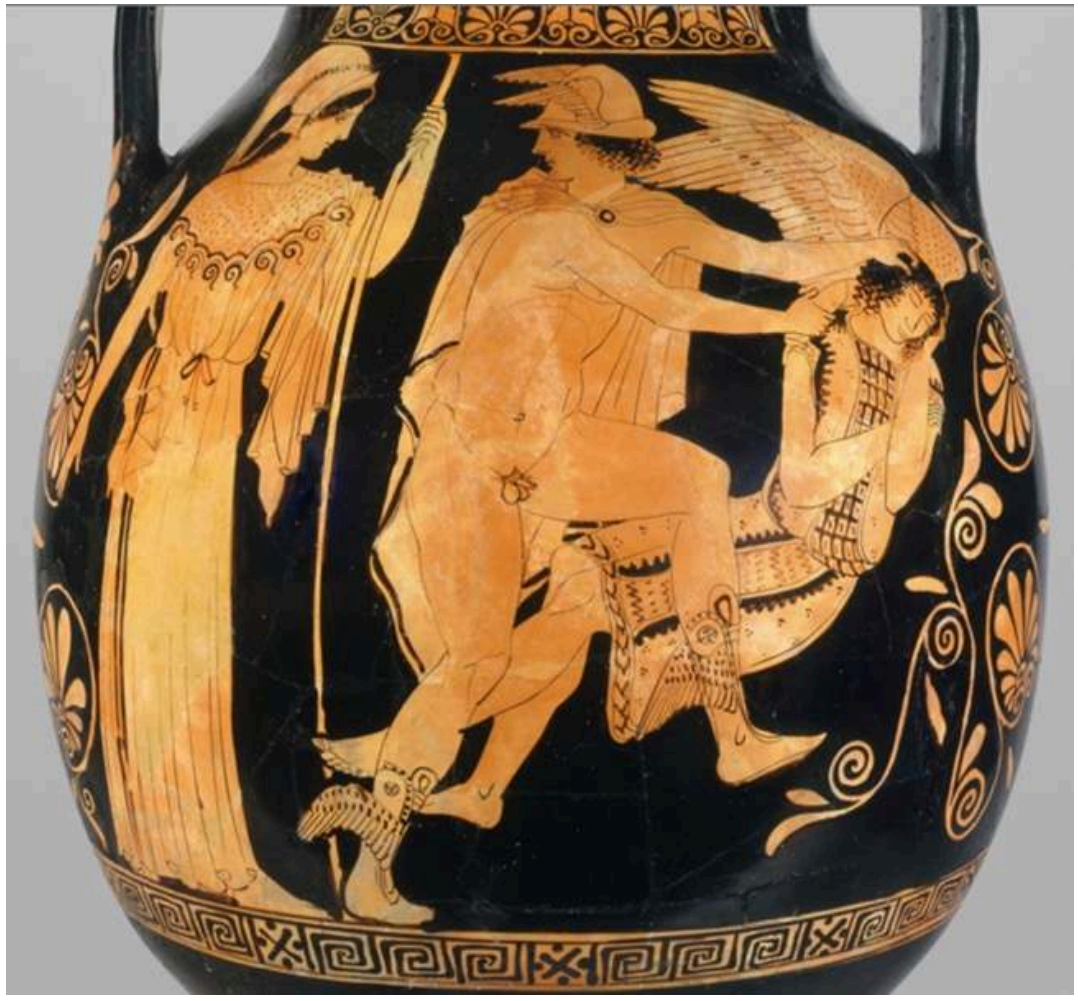

Péliké à figures rouges, New York, Metropolitan Museum of Art 45.11.1.

22 Ainsi la tête détournée au moment de la décapitation est réglementaire dès le $\mathrm{VII}^{\mathrm{e}}$ siècle sur le pithos à reliefs orientalisant en relief du viI ${ }^{\mathrm{e}}$ siècle ${ }^{15}$ (fig. 12) puis aux $\mathrm{VI}^{\mathrm{e}}$ et $\mathrm{V}^{\mathrm{e}}$ siècles sur l'olpé à figures noires ${ }^{16}$ (fig. 13) et en figures rouges sur la péliké ${ }^{17}$ (fig. 14) due à Polygnotos, où Méduse endormie a les yeux fermés. Le détournement du regard, complémentaire de la frontalité de la face de Méduse (devenue trois-quarts chez Polygnotos) construit une apostrophé figurative, signifiant que Méduse n'est visible que sous forme iconique. 
Figure 15

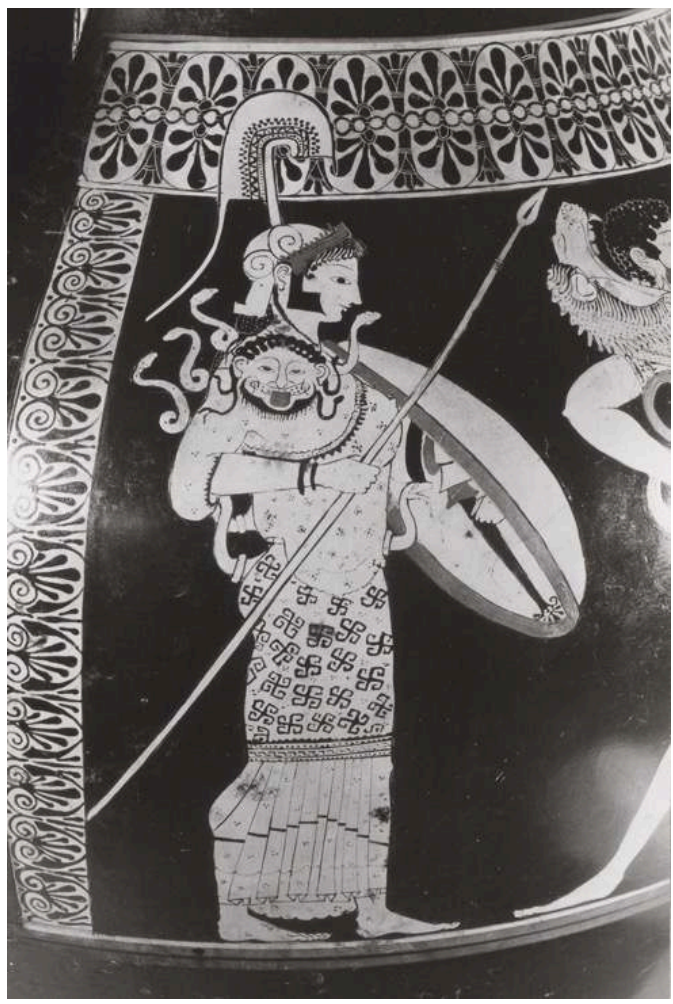

Amphore à figures rouges, Berlin, Antikensammlung F2159.

Figure 16

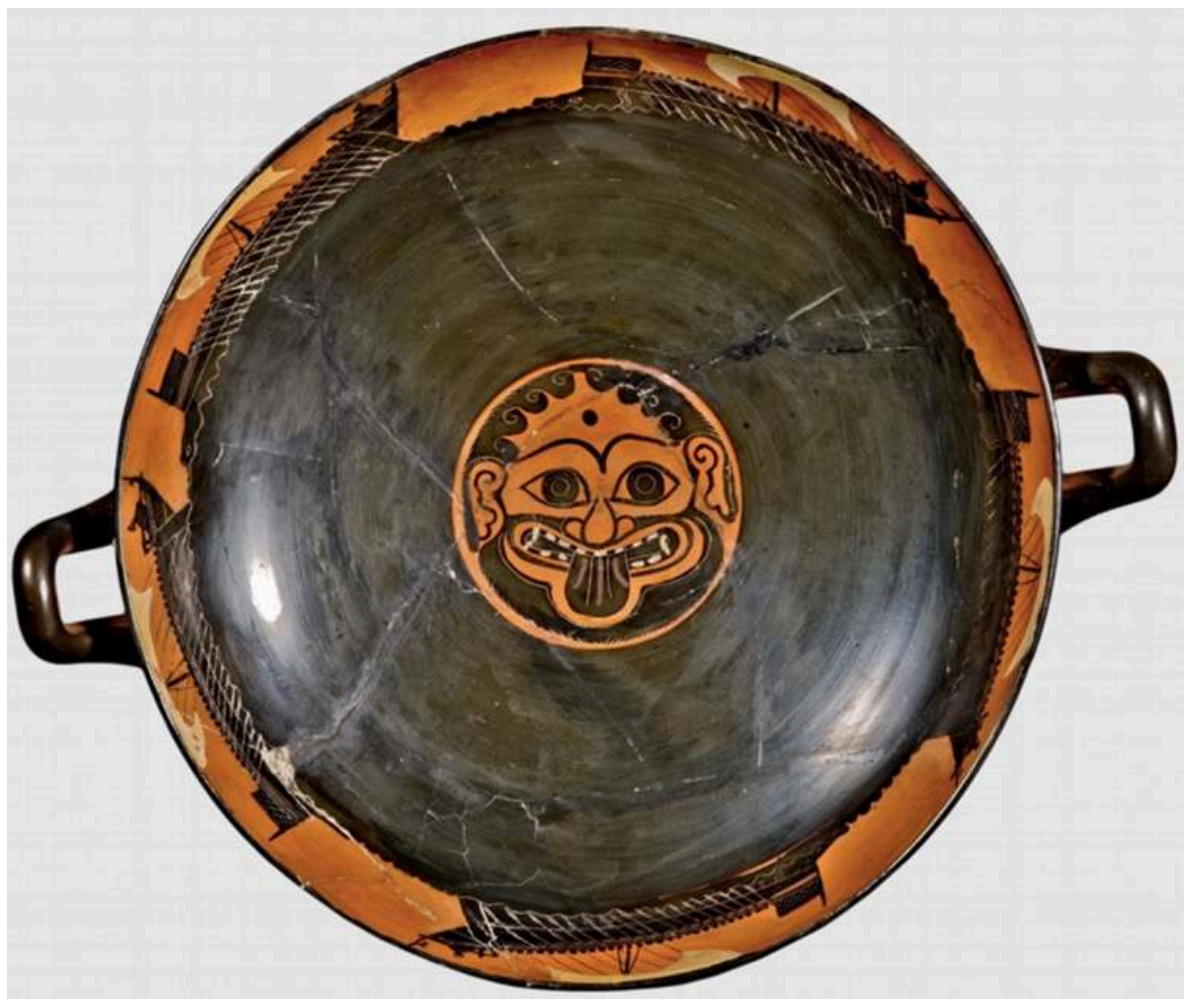

Coupe à figures noires, Paris, Cabinet des Médailles, cat. A. de Ridder 322. 
Parallèlement la figuration du gorgoneion oscille entre tête animée et image figée, que ce soit sur l'égide d'Athéna ${ }^{18}$ (fig. 15) ou en motif isolé décorant les coupes ${ }^{19}$ (fig. 16).

\section{Figure 17a}

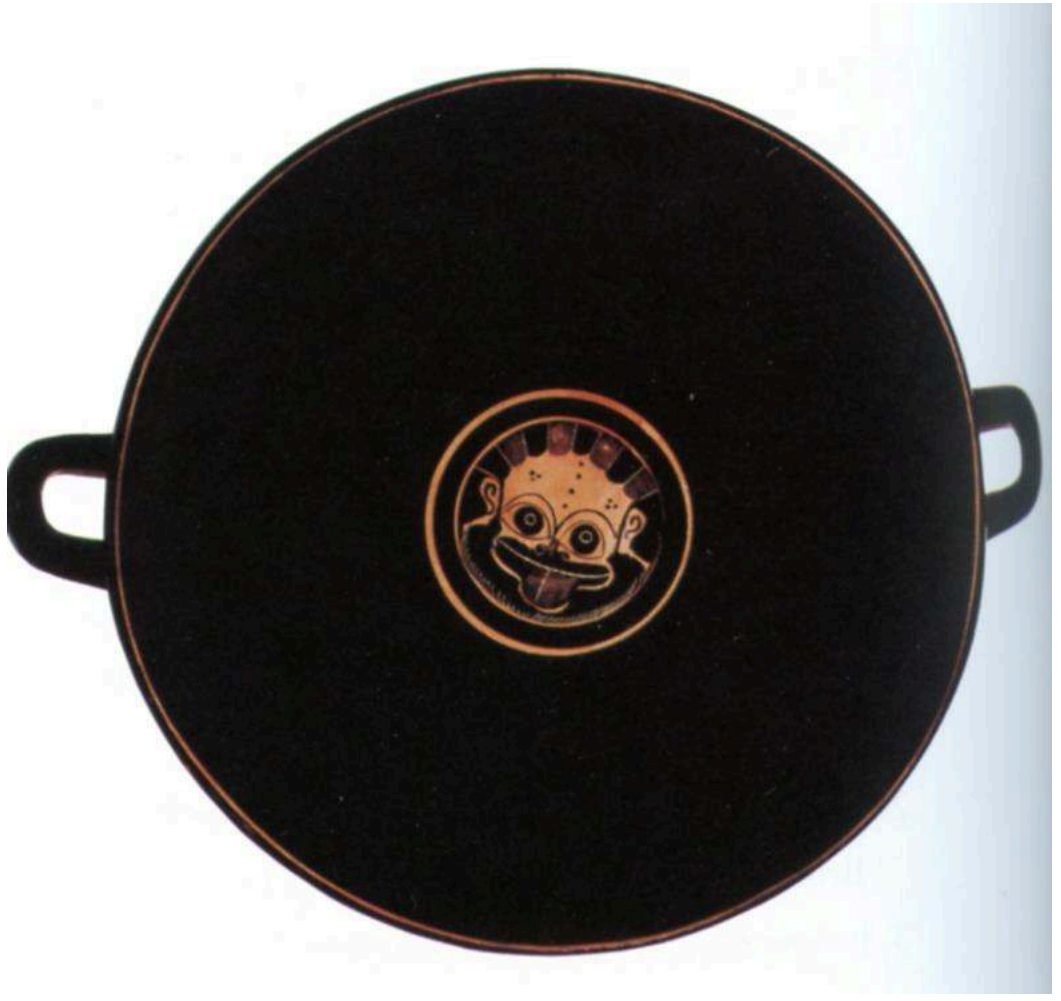

Coupe à figures noires, Madrid, Museo Arqueologico Nacional, coll. Varez Fisa 1999.99.71.

Figure 17b

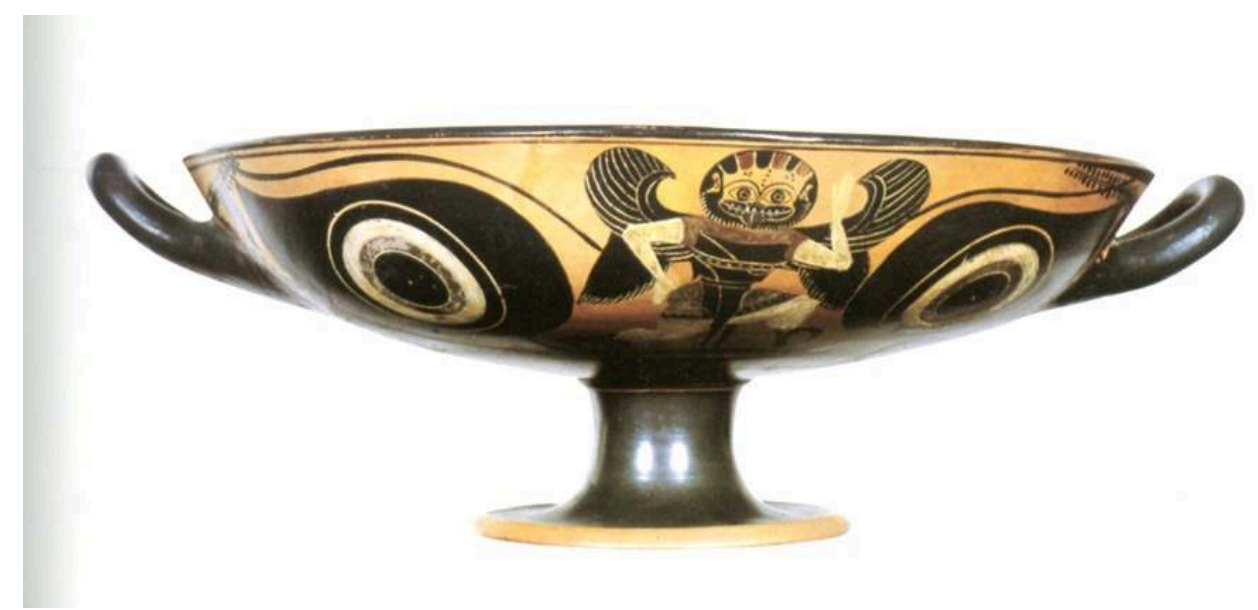

Coupe à figures noires, Madrid, Museo Arqueologico Nacional, coll. Varez Fisa 1999.99.71. 
Figure 17c
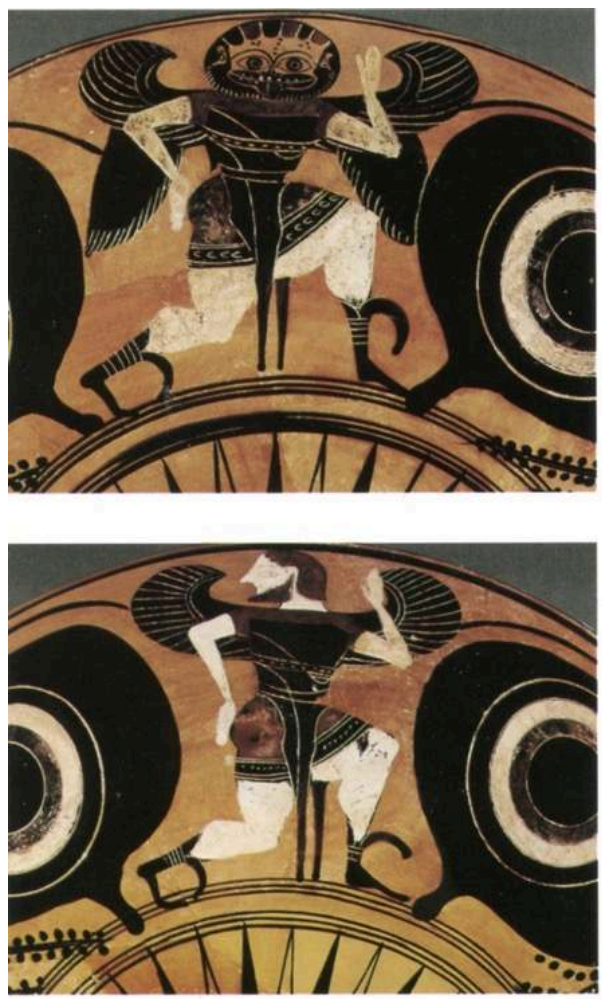

Coupe à figures noires, Madrid, Museo Arqueologico Nacional, coll. Varez Fisa 1999.99.71.

À cet égard une coupe de Madrid ${ }^{20}$ (fig. 17a, b, c) est éloquente : les deux faces externes se font écho. À Persée courant, tête retournée, répond une Gorgone intégrale, courant dans la même position, mais frontale. Elle est dotée, en lieu de tête, d'un gorgoneion bien cerclé, analogue à celui qui décore le médaillon interne.

Le gorgoneion, dérivé, tant sur le plan linguistique qu'au niveau figuratif, de la Gorgone, est particulièrement apte à exprimer le passage du modèle initial à ses succédanés en image.

\section{NOTES}

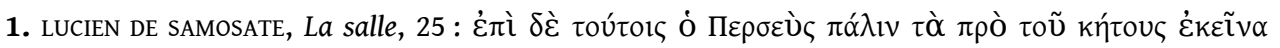

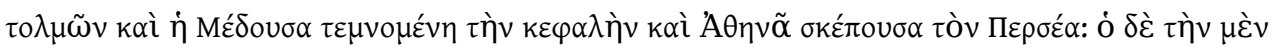

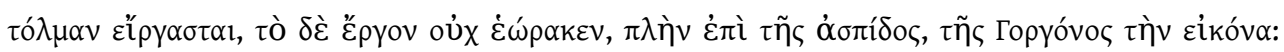

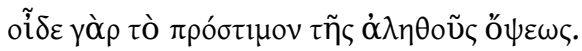

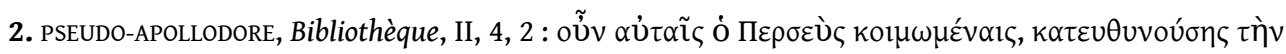

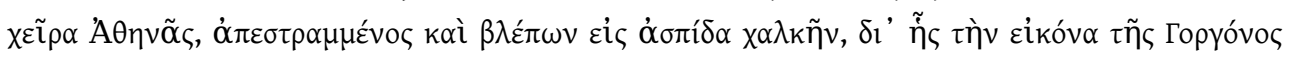

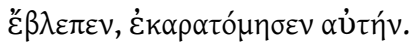

3. RVAp I 161.218 : vase connecté avec le peintre de Graz ; 380-360 av. J.-C. 
4. François LISSARRAGUE, La cité des satyres. Une anthropologie ludique (Athènes, $\mathrm{VI}^{e}-\mathrm{V}^{e}$ siècle avant J.-C.), Paris, éditions EHESS, 2013.

5. RVAp I 51.39 : peintre de Tarporley ; 400-375 av. J.-C.

6. RVAp I 48.16 : peintre de Tarporley ; 400-375 av. J.-C.

7. ABV 152.26 : Peintre d'Amasis ; 520-515 av. J.-C. ; BAPD 310453.

8. RVAp I 212.148 : peintre d'Ariane ; 400-375 av. J.-C.

9. RVAp I 51.44 : peintre de Tarporley ; 400-375 av. J.-C.

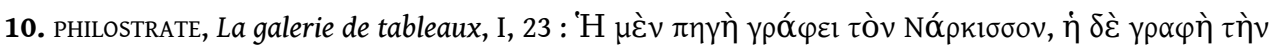

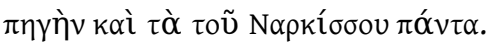

11. The Baleful Head (Cycle de Persée 8), 1886-1887, huile sur toile (155 x $130 \mathrm{~cm})$, Staatsgalerie Stuttgart inv. 3110.

12. ARV $^{2} 554.85$ : peintre de Pan ; 480-460 av. J.-C. BAPD 206328.

13. $A R V^{2} 555.96$ : peintre de Pan; vers 460 ; BAPD 206339.

14. $A R V^{2} 560.159$ : peintre de Pan, vers 460 av. J.-C. ; BAPD 206405.

15. Provenant de Béotie; vers 670 av. J.-C.

16. ABV 153.32 : peintre d'Amasis ; vers 550 av. J.-C. ; BAPD 310459.

17. $A R V^{2} 1032.55$ : Polygnotos ; 450-440 ; BAPD 213438.

18. $A R V^{2} 253.1$ : Peintre d'Andokides ; 520-500 av. J.-C. ; BAPD 200001.

19. ABV 380.296: Groupe de Léagros ; 510-500 av. J.-C. ; BAPD 302377.

20. Vers 520-510 av. J.-C. ; BAPD 9024720.

\section{RÉSUMÉS}

Comment représenter l'image d'une image insoutenable ? Justement, par une métaimage : un reflet, l'image d'une vision impossible (le regard de la Gorgone) dans l'image des peintres de vase et d'ekphrasis. L'article propose un parcours iconique entre textes (Lucien, Apollodore, Philostrate) et peintures de vases italiotes et attiques (en particulier celles du Peintre de Pan), sources narratives et réflexives, qui déclinent les stratégies adoptées "pour faire » voir enfin, autour de la décapitation de Méduse, le regard de la Gorgone.

How to represent the image of an unbearable image? Just by a metaimage: a reflection, the image of an impossible vision (the gaze of the Gorgon) in the image by the painters of vase and ekphrasis. This paper offers an iconic route between texts (Lucian, Apollodoros, Philostratus) and paintings of Italiot and Attic vases (in particular by the Pan Painter), as narrative and reflexive evidence, which decline available strategies to finally "let watch", around the beheading of Medusa, at the gaze of the Gorgon.

\section{INDEX}

Mots-clés : Gorgone, ekphrasis, Peintre de Pan, Céramique italiote, iconographie Keywords : Gorgon, ekphrasis, Pan Painter, South Italian ceramics, iconography 


\section{AUTEUR}

\section{FRANÇOISE FRONTISI-DUCROUX}

Françoise Frontisi-Ducroux est sous-directrice honoraire au Collège de France et membre du centre Anhima (anciennement au centre Gernet). Dans le mouvement de l'anthropologie historique de "l'école de Paris ", sa thèse de doctorat d'Etat, Prosopon. Valeurs grecques du masque et du visage, soutenue en 1987 à l'EHESS, a donné lieu à trois publications : Le Dieu-Masque (La Découverte, 1991), Du masque au visage (Flammarion, 1995, rééd. 2012) et « L'oeil et le miroir » in Dans l'oeil du miroir (Odile Jacob, 1997). Françoise Frontisi-Ducroux a consacré plusieurs autres ouvrages à la mythologie grecque, parmi lesquels L'Homme-cerf et la femme-araignée (Gallimard, 2003), Ouvrages de dames : Ariane, Hélène, Pénélope... (Seuil, 2009), Arbres filles et garçons fleurs : métamorphoses érotiques dans les mythes grecs (Seuil, 2017). 